\title{
Time resolved photoluminescence spectroscopy of surface-plasmon-enhanced light emission from conjugate polymers
}

\author{
Terrell D. Neal, ${ }^{a}$ Koichi Okamoto, and Axel Scherer \\ Department of Electrical Engineering, California Institute of Technology, MC 136-93, \\ Pasadena, California 91125-9300
}

\author{
Michelle S. Liu and Alex K.-Y. Jen \\ Department of Materials Science and Engineering, University of Washington, Box 352120, \\ Seattle, Washington 98195-2120
}

(Received 9 May 2006; accepted 23 October 2006; published online 28 November 2006)

\begin{abstract}
The authors have experimentally verified that the light emission from conjugated polymers can be enhanced through the use of surface plasmon coupling layers. Carrier dynamics of such plasmon-enhanced organic light emitters were studied and a recombination rate increase due to surface plasmon polaritons was experimentally observed. Internal quantum efficiency data from the polyfluorenes studied follow the trend supported by the time resolved photoluminescence measurements. (C) 2006 American Institute of Physics. [DOI: 10.1063/1.2397481]
\end{abstract}

Organic light emitting diodes (OLEDs) have become widely available and are used for replacing inorganic light emitting diodes as they are less expensive and provide many opportunities in regard to structural placement. Despite the tremendous promise for efficient solid state lighting offered by such organic light emitters, the road towards spectrally broad white light polymer emitters still holds many design challenges. Enhancement methods for efficient light emission have been studied for several inorganic semiconductors, ${ }^{1-3}$ and one very effective approach to developing highly efficient light emitters has been based on the incorporation of metals for the efficient extraction of light. ${ }^{4}$ Recently, we reported dramatic enhancements of the light emission from inorganic ${ }^{5,6}$ and organic ${ }^{7}$ light emitters using surface plasmon coupling. These can now be applied towards OLED material, as some already have, ${ }^{8,9}$ and incorporated together with advances in polymer chemistry that have recently resulted from the addition of fluorene based polymers or phosphors. ${ }^{10,11}$

Here we focus on increasing the light emission efficiency from optimized blue light emitting polymers through the use of surface plasmons for enhancement of the internal quantum efficiency. Three conjugated polymers were studied: PF-cyanophenylene(CNP) (1:1), PF-CNP (3:1), and polyfluorenes(PF)-triphenylamine(TPA)-quinoline(Q). The molecular structures for the conjugated polymers used are illustrated in Fig. 1. ${ }^{12,13}$ The corresponding conjugated polymers PF-CNP (1:1), PF-CNP (3:1), and PF-TPA-Q will be referred to as pf1cnp1, pf3cnp1, and pftpaq, respectively. The conjugated polymers were dissolved in chlorobenzene to form a $2 \%$ (by volume) solution. This solution was then spun onto a quartz sample, half of which had previously been coated with a $50 \mathrm{~nm}$ silver layer. Photoluminescence measurements were then performed to compare the different areas of the sample, comparing the luminescence efficiency from emitters with no metal with those in proximity to the metal.

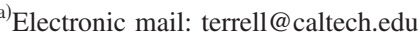

Similar to our earlier publication, ${ }^{7}$ the sample geometry consists of quartz samples covered with silver and light emitting polymer layers. In this letter, however, conjugated polymers are used instead of a dye doped polymer films, and we present photoluminescence results on these much more efficient emitter materials, in which the fluorophore concentration is higher compared to laser dyes doped within a polymer matrix. The organic light emitters presented here are similar in comparison with the state-of-the-art in terms of absolute efficiency based on a review of polyfluorenes presented by Neher, ${ }^{14}$ where polyfluorenes in solution and in solid state had photoluminescence quantum efficiencies around $50 \%$. More interesting observations are possible with such efficient polymer emitters.

The experimental setup and measurements for detecting emission intensity enhancements are described in our previous work ${ }^{7}$ on enhanced emission from dye doped polymer layers. An InGaN $405 \mathrm{~nm}$ diode laser was used to excite the samples. A Princeton Instruments cooled charge-coupled device and Acton Research Corporation spectrograph were

(a)

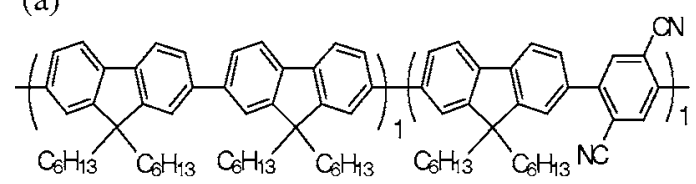

(b)
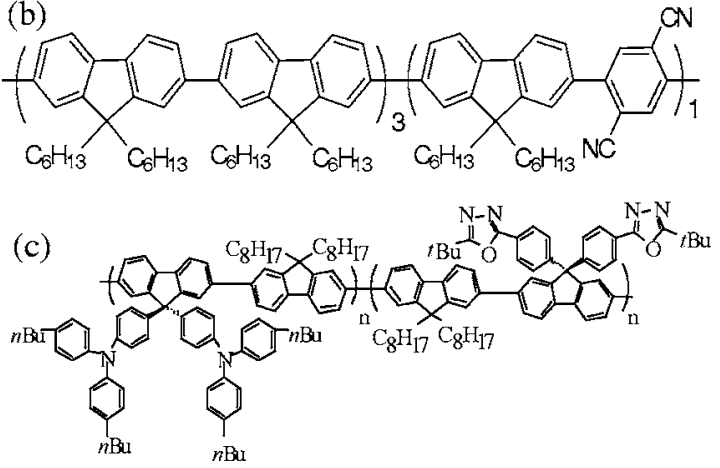

FIG. 1. Molecular structures for (a) pf1cnp1, (b) pf3cnp1, and (c) pftpaq 
(a)

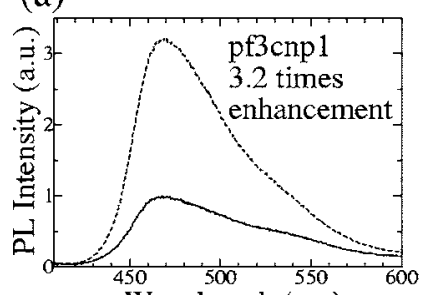

(c) Wavelength (nm)

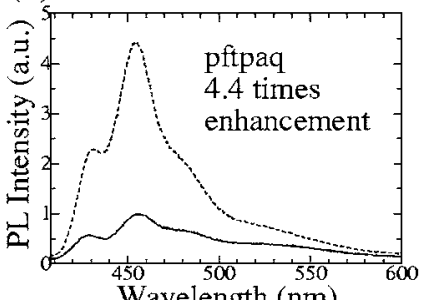

Wavelength (nm) (b)

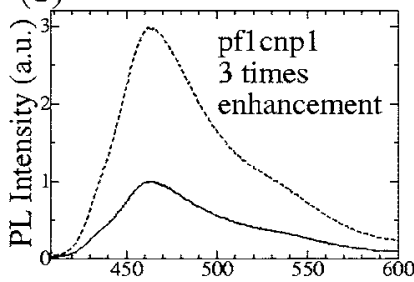

(d)

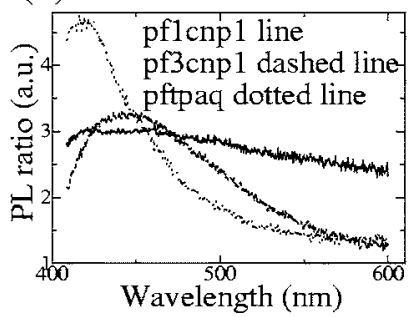

FIG. 2. PL enhancements for (a) pf1cnp1, (b) pf3cnp1, and (c) pftpaq on metal (dashed line) and without metal (solid line). (d) PL enhancement ratios plotted against the wavelength.

used to perform measurements of the emission intensities of these samples. Moreover, a Hamamatsu Photonics streak camera was used to obtain time resolved photoluminescence (TRPL) spectroscopy data from polyfluorene emission rates after deposition on silver coated quartz samples. For the TRPL measurements, a frequency doubled beam of a modelocked Ti:sapphire laser (Spectra Physics) was used to excite the samples. The pulse width, wavelength, and repetition rate were chosen as $100 \mathrm{fs}, 400 \mathrm{~nm}$, and $80 \mathrm{MHz}$, respectively. These TRPL studies determined decay rates at various wavelengths, as well as the Purcell ${ }^{15}$ factors. Carrier dynamic measurements were observed to be correlated with measured photoluminescence intensity enhancement ratios and confirm that some conjugate polymers are better suited for luminescence enhancement resulting from coupling to surface plasmons. A neutral density filter was added in the setup just before the detector as the signal intensities were significantly larger than from our previous dye doped polymer work. By employing the use of a shutter, photo-oxidation of these somewhat unstable conjugated polymers could be largely avoided.

The enhancements observed for pf $1 \mathrm{cnp} 1$, pf $3 \mathrm{cnp} 1$, and pftpaq were 3, 3.2, and 4.4 times, respectively, and are shown in Figs. 2(a)-2(c). In Fig. 2(d) the enhancement ratios for each conjugated polymer reflect a maximum enhancement for shorter wavelengths. A larger contribution due the surface plasmon coupling is evident as shorter wavelengths have higher enhancements. This finding can be explained by the surface plasmon dispersion diagram, which indicates a better match between the silver plasmon resonance and the emission at shorter wavelengths.

The emission enhancement of the conjugated polymer on Ag sample can be attributed to excited conjugated polymer molecules coupling to the electron vibration energy of surface plasmon (SP). As illustrated in Fig. 3(a), a SP instead of a photon is produced by the molecular relaxation processes and thereby leads to an increase in the spontaneous recombination rate. This coupling mechanism resembles one presented for the inorganic semiconductor case, ${ }^{16}$ although there is no buffer or cap layers as the conjugated polymer can be placed directly on the metal. When the conjugated can be placed directly on the metal. When the conjugated shown below.
Downloaded 06 Dec 2006 to 131.215 .250 .40 . Redistribution subject to AIP license or

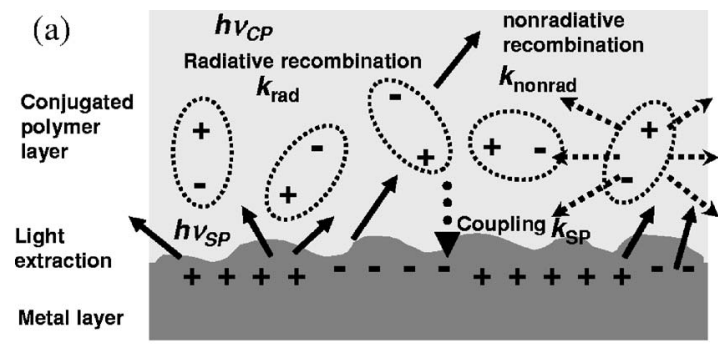

(b)

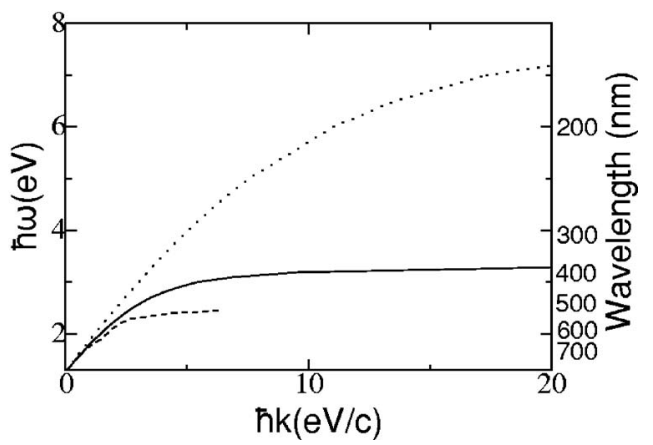

FIG. 3. (a) Underlying physical mechanism cartoon depiction. (b) Surface plasmon dispersion diagram, polymer interfaced with $\mathrm{Au}$ (dashed line), Ag (solid line), and $\mathrm{Al}$ (dotted line).

polymer is not adjacent to a rough metal film, these excited conjugated polymer molecules are terminated by the radiative $\left(k_{\text {rad }}\right)$ or nonradiative $\left(k_{\text {nonrad }}\right)$ recombination rates. For samples where the conjugated polymer is on the rough metal film, the energy of the conjugated polymer $\left(h v_{\mathrm{CP}}\right)$ obtained by way of photopumping or electrical pumping is close to the electron vibration energy $\left(h v_{\mathrm{SP}}\right)$ of the SP at the metalsemiconducting polymer interface, then the energy can transfer to the SP, with coupling rate $\left(k_{\mathrm{SP}}\right)$. The metal film roughness allows for SPs of high momentum to scatter, lose momentum, and couple to radiate light. ${ }^{17}$

Large density of states, evident on the SP dispersion diagram in Fig. 3(b), introduces high electromagnetic fields which increase $k_{\mathrm{SP}}$. We note also in this figure that this surface plasmon enhancement method can be applied in the UV and across the visible range, depending on the metal chosen, where aluminum is adequate for UV, silver for the blue region, and gold for the red region.

Figures 4(a)-4(c) compare the time resolved PL decay profiles between the conjugated polymers deposited on bare quartz and silver coated quartz sample. Longer PL lifetimes were measured for the conjugated polymer on bare quartz. The observation of a faster decay profile for pf1cnp1 on metal on quartz suggested that there was a significant transfer of energy between the conjugated polymer and the silver surface plasmon polariton. Conjugated polymers with bipolar charge-transporting properties, namely, pf $3 \mathrm{cnp} 1$ and pftpaq, already exhibited fast decay rates without the silver layers and therefore the lifetime is similar to that when surface plasmons are coupled, in turn providing no significant reduction in the PL lifetime, $\tau_{\mathrm{PL}}$. However, for the better electron transporter pf1cnp1, energy transfer is facilitated to the surface plasmons and results in shorter PL lifetimes.

The internal quantum efficiency $\eta_{\text {int }}$ is described as the radiative recombination rate divided by the sum of the radiative $k_{\text {rad }}$ and nonradiative recombination rates $k_{\text {nonrad }}$ as

to AlP license or copyright, see http://apl.aip.org/apl/copyright.jsp 

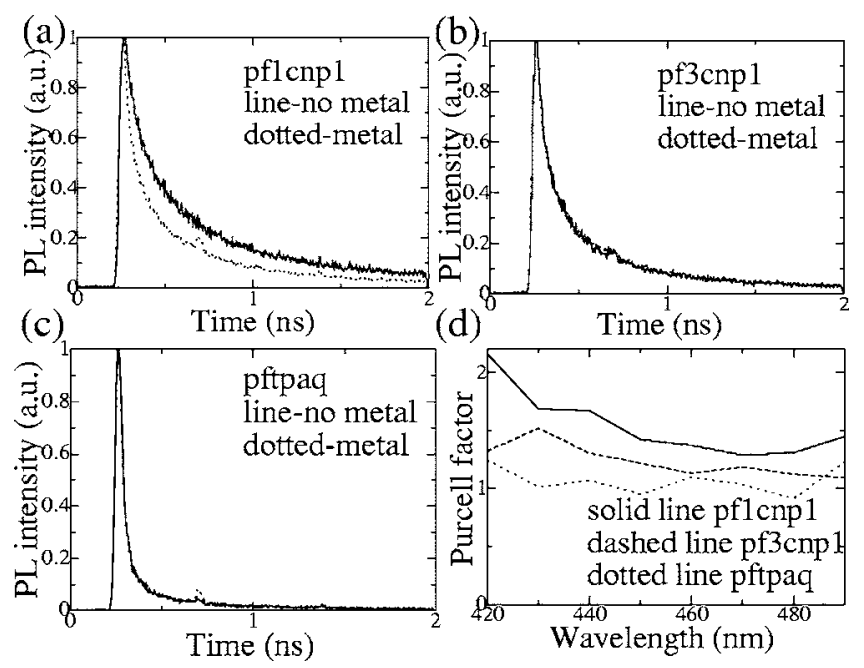

(d)

Time (ns)

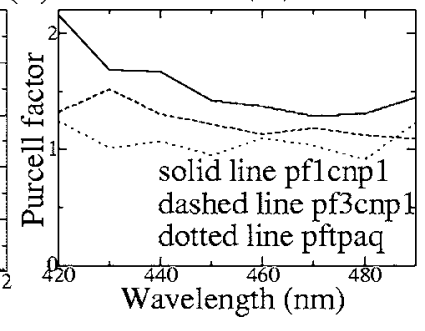

FIG. 4. TRPL with the data normalized to 1 where the dotted line show the conjugated polymer carrier decay rate with metal and solid line without metal, (a) pf1cnp1, (b) pf3cnp1, and (c) pftpaq. (d) Average Purcell factor, comparing the carrier lifetime, plotted against the wavelength for the conjugated polymers studied.

$$
\eta_{\text {int }}=\frac{k_{\text {rad }}}{k_{\text {rad }}+k_{\text {nonrad }}},
$$

where the PL lifetime is the inverse of the recombination rate (i.e., $1 / \tau_{\mathrm{PL}}=k_{\mathrm{rad}}+k_{\mathrm{nonrad}}$ ). For the surface plasmon case, the enhanced efficiency, $\eta_{\text {int }}^{*}$, is better described by the sum of the recombination rates that are radiative and due to surface plasmons, $k_{\mathrm{SP}}$, divided by the sum of the radiative, nonradiative, and surface plasmon recombination rates, as listed below. ${ }^{5,6}$

$$
\eta_{\text {int }}^{*}=\frac{k_{\text {rad }}+k_{\mathrm{SP}}}{k_{\mathrm{rad}}+k_{\text {nonrad }}+k_{\mathrm{SP}}} .
$$

If initially the efficiency is high, the influence from the surface plasmons is not significant. The coupling due to surface plasmons can become very significant when the initial quantum efficiency is low.

In Fig. 4(d), the ratio of the photoluminescence lifetimes is calculated and represented as the Purcell factors plotted versus the emission wavelengths for the three conjugated polymers. Carrier lifetimes are shorter for the case when the conjugated polymer was deposited on the metal films. These Purcell factors represent the average of several measurements taken for each conjugated polymer. Again, the pf1cnp1 sample showed the greatest influence on the metal surface. When the initial lifetime was short, coupling into surface plasmons did not significantly decrease the lifetime. For the case when the initial lifetime was long, however, surface plasmons could substantially decrease the lifetime and increase the quantum efficiency.

Taking an in-depth look at the properties of the polyfluorenes studied can reveal the mechanisms that offer themselves to better coupling potential. A high efficiency due to better charge injection and more efficient charge recombination was reported by Shu et al. for PF-TPA-Q. ${ }^{13}$ Liu et al. extensively studied such properties of the PF-CNP (3:1) and
PF-CNP (1:1), where the solid state PL efficiencies were reported as $66 \%$ and $48 \%$, respectively. ${ }^{12}$ They also attributed the slow electron motion to the higher content of cyano constituents that stabilize the radical anion and the less planar structure of PF-CNP (1:1). For these reasons, we expect the surface plasmon polaritons to assist in the movement of these electrons, coupling enough energy to increase electron transport.

Some enhancement due to the coupling of surface plasmons was evident in the PL data obtained from the metal side. These enhancements, however, were not as significant as for the dye doped polymer layers. We attribute this observation to the notion that the efficiencies of the conjugated polymers are already high and we expect the coupling due to surface plasmons to have a smaller effect on the enhancement in this case.

From studying the TRPL of these polyfluorenes in conjunction with the PL enhancement ratios we have found that those with longer carrier lifetimes offer large improvements in quantum efficiency if surface plasmons are used to enhance recombination of electron-hole pairs. Although only three or four times emission enhancements are observed this is a significant increase in quantum efficiency from these state-of-the-art organic light emitters. We expect that, along with the development of robust and efficient polymers, the use of surface plasmons will ultimately lead to highly efficient polymer light emitters that can be used for solid state lighting applications.

One of the authors (T.D.N.) acknowledges support from Center for Materials and Devices and Information Technology Research Fellowship. This work was also supported by the NSF Science and Technology Center under Grant No. DMR-0120967 and by DARPA under Grant No. HR0011-04-1-0032.

${ }^{1}$ I. Gontijo, M. Borodisky, E. Yablonovitch, S. Keller, U. K. Mishra, and S. P. DenBaars, Phys. Rev. B 60, 11564 (1999).

${ }^{2}$ A. Neogi, C.-W. Lee, H. O. Everitt, T. Kuroda, A. Tackeuchi, and E. Yablonovitch, Phys. Rev. B 66, 153305 (2002).

${ }^{3}$ N. E. Hecker, R. A. Hopfel, N. Sawaki, and T. Maier, Appl. Phys. Lett. 75, 1577 (1999).

${ }^{4}$ J. Vuckovic, M. Loncar, and A. Scherer, IEEE J. Quantum Electron. 36, 1131 (2000).

${ }^{5}$ K. Okamoto, I. Niki, A. Scherer, Y. Narukawa, T. Mukai, and Y. Kawakami, Appl. Phys. Lett. 87, 071102 (2005).

${ }^{6}$ K. Okamoto, I. Niki, A. Shvartser, Y. Narukawa, T. Mukai, and A. Scherer, Nat. Mater. 3, 601 (2004).

${ }^{7}$ T. D. Neal, K. Okamoto, and A. Scherer, Opt. Express 13, 5522 (2005).

${ }^{8}$ P. Andrew and W. L. Barnes, Science 306, 1002 (2004).

${ }^{9}$ P. A. Hobson, S. Wedge, J. A. E. Wasey, I. Sage, and W. L. Barnes, Adv. Mater. (Weinheim, Ger.) 14, 1393 (2002).

${ }^{10}$ F. Hide, P. Kozodoy, S. P. DenBaars, and A. J. Heeger, Appl. Phys. Lett. 70, 2664 (1997).

${ }^{11}$ C. Zhang and A. J. Heeger, J. Appl. Phys. 84, 1579 (1998).

${ }^{12}$ M. S. Liu, X. Jiang, P. Herguth, and A. K.-Y. Jen, Chem. Mater. 13, 3820 (2001).

${ }^{13}$ C.-F. Shu, R. Dodda, F.-I. Wu, M. S. Liu, and A. K.-Y. Jen, Macromolecules 36, 6698 (2003).

${ }^{14}$ D. Neher, Macromol. Rapid Commun. 22, 1365 (2001).

${ }^{15}$ E. M. Purcell, Phys. Rev. 69, 681 (1946).

${ }^{16}$ K. Okamoto, I. Niki, A. Scherer, Y. Narukawa, T. Mukai, and Y. Kawakami, Appl. Phys. Lett. 87, 071102 (2005).

${ }^{17}$ W. Barnes, Nat. Mater. 3, 588 (2004). 